\title{
Effects of the imposter phenomenon on measures of assertiveness in female professionals in South Africa
}

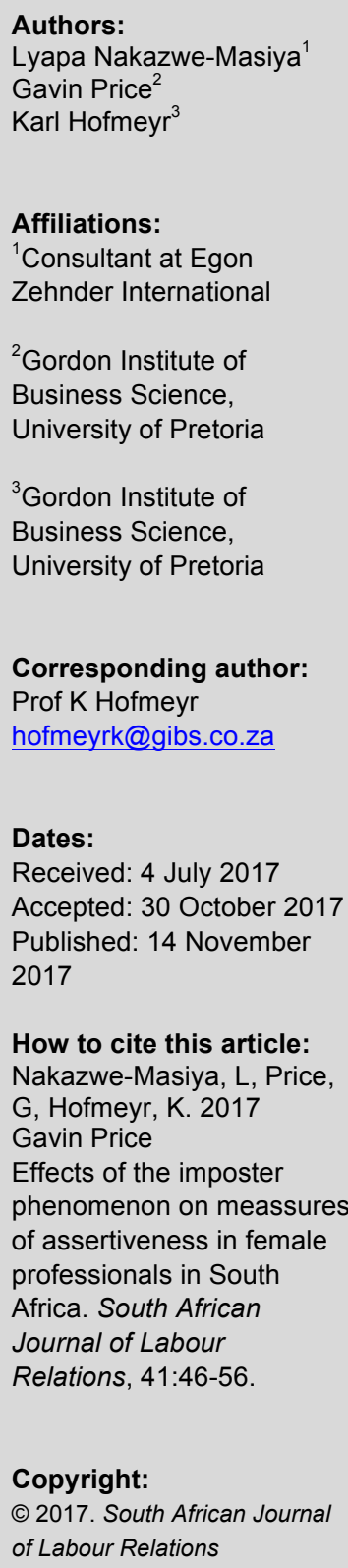

\begin{abstract}
Assertiveness is arguably one of the strongest predictors of success among business leaders and professionals. The study focuses on imposter phenomenon (IP), which occurs when an individual, normally considered to be a high achiever, undergoes an internal experience of feeling like an intellectual fraud or experiences a fear of failure upon achieving success, and the relationship of IP with assertiveness. Due to an absence of research on the relationship between IP and assertiveness, particularly in professionals in South Africa, this study investigates the way these two variables relate to each other. The purpose of this study is to 1) investigate whether IP is prevalent in female professionals in South Africa, 2) investigate whether IP and assertiveness are correlated and 3) determine whether IP is a predictor of assertiveness. The study was conducted on working professionals in South Africa $(\mathrm{N}=165)$ and questionnaires were distributed online. The instruments used were the Clance Imposter Phenomenon Survey and elements of the Adaptive and Aggressive Assertiveness Scales. The professionals assessed were drawn from different industry sectors and across all job functions. The results suggest that IP is prevalent in professionals in South Africa and bears a moderately negative relationship to assertiveness. Another finding was that IP is a negative predictor of assertiveness. Gender also had an effect on the relationship between the two variables; the relationship was only found to be significant among women when the sample was split.
\end{abstract}

Keywords: assertiveness, imposter phenomenon, leadership, gender

\section{Introduction and background}

"Look like a girl, act like a lady, think like a man, work like a boss" - in the words of a 2015 press release by BIC, a South African company that makes pens. This was published on their Facebook page during women's month, with the intention of empowering women (Eleftheriou-Smith 2015). The statement caused an uproar and resulted in BIC issuing a public apology. The reality though is that many women are operating in a maledominated world and are expected to behave like their male counterparts (Howe-Walsh \& Turnbull 2016). Women in management roles often lack assertiveness as they have been conditioned to be passive and operate in non-assertive roles (Viswanatham \& Sharma 2013). According to Pfafman and McEwan (2014), women in the workplace are faced with the conundrum of being considered either too feminine to be professional or too professional to be feminine. Women therefore face a constant dilemma as they endeavour to manage their assertiveness in the workplace (Howe-Walsh \& Turnbull 2016).

Viswanatham and Sharma (2013) propose that female professionals with higher levels of assertiveness are likely to have greater expectations of achievement and better coping mechanisms in their personal and work lives. Their study also revealed that female professionals who lack assertiveness tend to have a negative outook and are not inspired to progress or strive for achievement.

Various reasons have been advanced as to why women leaders often lack assertiveness, including fear of failure, a dimension which is closely associated with the imposter phenomenon or syndrome (Clance \& Imes 1978). Clance and Imes (1978) coined the term imposter phenomenon and describe IP as a common experience that occurs when an individual, normally considered to be a high achiever, undergoes an internal 
experience of self-doubt and feels like an intellectual fraud or suffers fear of failure upon reaching milestones or achieving success. Neureiter and Traut-Mattausch (2016) suggest that as long as overachievers can prevent themselves from being successful, they will limit their chances of feeling like a fraud and in turn avoid facing rejection.

Our study set out to determine whether there is indeed a relationship between IP and levels of assertiveness in female professionals in the South African context. Many managerial environments are skewed towards male managers and leaders and if there is a trend towards transformational leadership with less "command and control", women should be well placed to take their rightful place alongside men in the leadership ranks (Powell 2012).

\subsection{Evidence}

A report dealing with female leadership in South Africa showed that only $8.79 \%$ of the large companies listed on the Johannesburg Stock Exchange had more than $25 \%$ females in directorship positions (Mall 2015). The report argued that South Africa would not be able to compete on the international stage if it was unable to develop and retain high-calibre female talent. Having female representation on boards and in professional roles has been on the agenda for business globally and continues to be a challenge (Mall 2015).

In looking for reasons for the slow advancement of women into leadership positions, a number of studies have shown that leadership ineffectiveness is often associated with a lack of assertiveness (Ames \& Flynn 2007). Moreover, the Trait Theory of leadership states that leaders are likely to display various qualities such as extraversion, which encompasses assertiveness and enthusiasm, agreeableness and openness (DeYoung, Weisberg, Quilty \& Peterson 2013). Our study set out to investigate whether IP is also a factor that negatively influences levels of assertiveness in female professionals in South Africa, thus impacting on their leadership effectiveness.

Organisations are inherently gender-biased as a result of being created for and by men. The social practices are often subtle and insidious and disadvantage women, thus compromising their ability to be effective in the workplace (Ely \& Meyerson 2000).

\subsection{Business relevance}

A recent McKinsey study proposed that if women played an identical role to men in the global labour market and economy, as much as $28 \%$ could be added to the global GDP by the year 2025 (Woetzel 2015). The need for diversity in the workplace at senior management levels is a global challenge that has to be resolved by ensuring that women are being developed and equipped with the relevant skills and experience to ensure that they become effective leaders (Woetzel 2015). One element of this relates to assertiveness: if women are more assertive, it is expected that they will become more effective as leaders (Cook \& Glass 2014).

\subsection{Motivation for the study}

The imposter phenomenon has been shown to hamper career growth in both males and females. In a study conducted by Vergauwe, Wille, Fey, De Fruyt and Anseel (2015), it was shown that IP can prevent professionals from progressing to more senior managerial roles. Research on the topic has been conducted in various geographic regions over the last 40 years, in particular Europe, Asia and America (Sakulku \& Alexander 2011; Peteet, Brown, Lige \& Lanaway 2015; Kwan 2015). Limited research has been conducted in Africa and therefore this study aimed to enhance the literature on IP from an African perspective, in particular from the perspective of South Africa.

Ames and Flynn (2007) argue that leaders normally display three levels of assertiveness: low (e.g. being reflexive and polite to others), moderate (e.g. growing defensive as and when provoked), and high (e.g. seeking situations that result in hostility). Their study revealed that reasonable levels of assertiveness were expected to result in effective leadership, in terms of both work and social outcomes. However, in many cases female leaders may have been conditioned by society to be passive and to take on roles that do not require them to be assertive (Viswanatham \& Sharma 2013).

From a business perspective, there is a need to discover why females are not fully represented at leadership levels. It is necessary to establish whether assertiveness is something females need to work on in order to progress. Howe-Walsh and Turnbull (2016) suggest that IP may be a barrrier to women leaders' attainment of more senior roles. Our paper investigates whether there is indeed a relationship between assertiveness and IP and whether the relationship is affected by the gender of the manager concerned.

\section{Literature review}

Clance and Imes's (1978) study found that despite the 150 women in their study having earned degrees, educational honours, high results on standardised assessments, affirmation and professional 
acknowledgement from colleagues and respected authorities, the women surveyed did not have an internal sense of success. The women interviewed who experienced IP attributed their success to luck, charm or a temporary internal cause such as effort, rather than to their ability. Despite experiencing selfdoubt, they nevertheless reported a strong desire to look intelligent and to be the best among their peers.

Studies now suggest that the imposter phenomenon can affect both men and women in occupations that differ in subjective difficulty and status (e.g., Arena \& Page 1992; Bernat 2008; Clark, Vardeman \& Barba 2014; Fried-Buchalter 1997; Mattie, Gietzen, Davis \& Prata 2008).

The theory of IP was later expanded by Clance, Dingman, Reviere and Stober (1995), who state that when imposters, individuals with significant IP traits, are unable to appreciate their talents and competencies, they compare their strengths and weaknesses with those of others and undermine their talents and competencies. Imposters tend to avoid intellectual challenges as they struggle with the notion of maintaining the façade of success. Cokley, McClain, Enciso and Martinez (2013) note that imposters find it difficult to accept their accomplishments and are hesitant to attribute their victories to inherent skills or intelligence. Despite peripheral proof, these imposters attribute their achievements to transient causes such as coincidence, mistake, or diligence. Schubert and Bowker (2017) have found people with low self-esteem to be particularly vulnerable to feeling that they are imposters. The imposter phenomenon has been recognised as a barrier to entry and progression for women in male-dominated fields such as science, technology, engineering and mathematics (Linderman, Britton \& Zundi 2016; Simmons 2016).

\subsection{Dimensions of IP}

Clance (1985) identified six dimensions of IP: the imposter cycle, the need to be the best, superhuman attributes, the fear of failure, denial of competence and discounting praise, and fear and guilt regarding success. According to Dudău (2014), imposters normally react in one of two ways: either they overcompensate by putting in an extreme effort or they engage in self-sabotage activities such as procrastination. If by chance imposters are over-prepared, they convince themselves that their success has been due to hard work. Impostors strongly believe that their achievements gained through hard work are not a reflection of true or genuine capabilities (Clance 1985). As observed by Sakulku and Alexander (2011), imposters were often high achievers throughout their early lives and feel the need to carry this on as they grow older. Imposters tend to set impossible and sometimes unachievable targets for themselves and when unable to achieve these, they see this as a sign of failure. Clance (1985) describes this characteristic as superwoman and superman. Imposters believe that when they achieve success they will be isolated and rejected by their peers. Sakulku and Alexander (2011) conclude that the fear is not about success leading to rejection, but rather that imposters also fear that their success will result in greater demands and higher expectations from others around them.

\subsection{Antecedents of IP}

Research suggests that there are a number of causes of IP, ranging from family origins to social origins, which raises the question whether IP is a matter of nurture or nature. Langford and Clance (1993) state that in terms of family origins, IP may be brought about by childhood experiences where a child is only occasionally validated and is normally unsupported by a family that is often in conflict and without channels of expression. The child then works hard in order to seek approval and recognition.

It can be argued that some females, minorities and other marginalised individuals are socialised to expect that they will not advance at the same rate as their male counterparts. Clance et al (1995) discovered that these individuals feel that they are frauds as they progress up the hierarchies in which they feel they do not belong. Women are susceptible to imposter feelings because society does not expect them to succeed. Women report that they lack the social support that is offered to males and often worry that if they are successful in their careers it will compromise their feminine characteristics (Li, Hughes \& Thu 2014). Achievement-oriented behaviours that imposters engage in are driven by the desire to be considered intelligent by their peers and shaped by a dominant concern as to what impression they leave with others (Langford \& Clance 1993).

\subsection{IP in relation to gender}

Early studies suggested that IP was more prominent in females (Clance \& Imes 1978; Langford \& Clance 1993). These studies show that IP has a bigger effect on women as they are affected by a constant battle between feminine and masculine traits such as autonomy. Cusack, Hughes and Nuhu (2013) in their investigations of IP, mental health and gender, also argue that females are more prone to IP. They attribute this to the demands that women face as a result of the multiple roles they play in society, and the societal pressure on them to be "superwomen". 


\subsection{IP and leadership}

Neureiter and Traut-Mattausch (2016) conclude that IP functions as an inner barrier to moving up to higher occupational levels and leadership positions. Their study showed that IP is negatively related to work and employee outcomes. Imposters often feel insecure and consequently find it difficult to progress in their organisations. They also found that students with IP are reluctant to take on senior leadership roles. The study by McDowell, Grubb and Geho (2015) found that respondents from their samples who had IP traits exhibited low levels of self-efficacy and this resulted in issues such as fatigue, anxiety and burnout in the workplace, despite their obvious skills and abilities.

\subsection{Assertiveness}

This study investigates the relationship between IP and assertiveness and the extent to which these factors affect the advancement of women at work. Stein and Book (2010) describe assertiveness as an element of emotional intelligence: an assertive personality enables one not only to undertake emotional reflection, which facilitates the understanding of one's own feelings and emotions, but also to manage personal impulses, thereby exhibiting self-control and behaving in an appropriate manner and with appropriate dynamism.

Ames and Flynn's (2007) research found that leadership development and effectiveness are positively associated with assertiveness. Lazarus (1973) identifies four behaviours of assertive people, namely the ability to communicate openly about their needs and desires, to say "No", to freely discuss their feelings, and to form networks, as well as to initiate, maintain and conclude conversations.

Assertive behaviours and skills have been shown to be beneficial, particularly in business relationships. Assertive people are considered powerful in comparison with their passive colleagues and as such, they tend to adopt more senior roles based on their behaviour and social networks (Ames \& Flynn 2007). Lounsbury, Sundstrom, Gibson, Loveland and Drost (2016) found in their study on personality traits of managers that successful managers display high levels of assertiveness along with extraversion, work drive and emotional stability.

\subsection{Gender and assertiveness}

Rohrmann, Bechtoldt and Leonhardt (2016) found that females in leadership roles have characteristics that are normally associated with masculine personality traits such as assertiveness, determination and confidence. Their findings suggest that these personas are developed to enable females to meet the everyday challenges and expectations of being in male-dominated professional roles. Assertive female leaders have been found to be suitable leaders in a team setting owing to their ability to voice their opinions as well as act with the team goal and interests in mind without experiencing elements of selfdoubt or insecurity (Parham, Lewis, Fretwell, Irwin \& Schrimsher 2015).

Women are generally associated with compassionate traits when relating to others. These traits include being warm, accommodating, welcoming, gentle and understanding, along with showing consideration and calmness. Conversely, men tend to display agentic qualities, such as dominance, assertion and control (Eagly \& Carli 2007). Braidford, Stone and Tesfaye (2013) suggest that in many cases, women leaders feel the need to take on masculine traits in order for them to perform and think like managers, and they adapt their management styles accordingly. The stereotyped way in which women are assessed is argued by Heilman (2001) to be a key reason for their failure to progress up the corporate ladder. Rossette, Koval, Ma and Livingston (2016) propose a framework that sets out the dimensions and nature of the stereotyping and biases that face women of different races. They postulate that awareness of these dimensions may assist in overcoming the challenges women face in processing to leadership positions. Organisational cultures are not genderless and women who enter male environments are required to assume male patterns of behaviour while at the same time preserving their female characteristics (Gherardi \& Poggio 2001).

To date little research has been conducted on IP and its relationship with assertiveness in the South African context. Research conducted internationally has shown that females are more prone to IP than men and it appears to be more prevalent in minority groups. This study seeks to establish whether IP in females affects their assertiveness.

\section{Research question and hypotheses}

This research firstly sought to answer the question whether or not IP is indeed prevalent among professionals in South Africa. It then aimed to establish whether IP negatively influences assertiveness, particularly among female professionals in South Africa. To this end, two hypotheses were developed for testing: 


\subsection{Hypothesis 1}

Building on the literature, the first hypothesis developed for testing in this study was that there is a negative correlation between IP and assertiveness and further that IP is a negative predictor of assertiveness.

\subsection{Hypothesis 2}

IP has a greater effect on female professionals than on male professionals.

\section{Methodology}

Quantitative research involving the collection of primary data was conducted with a sample of respondents that was selected on the basis of its ability to provide a source of professional men and women working in South Africa. Respondents were considered to be professionals if they worked as a knowledge worker, managed others, or managed functions, business units or entities. A non-probability convenience sample was therefore used in the current study to maximise the number of responses within the time and financial constraints of the study (Saunders, Lewis \& Thornhill 2012). They were considered representative of the population for the study, namely professionals who are employed in a full-time capacity in South Africa.

\subsection{Measurement instrument and pilot study}

The measurement instrument employed in the study was a self-reporting questionnaire that consisted of three sections, Clance's Imposter Scale (CIPS), six questions adapted from the Adaptive and Aggressive Assertiveness Scale (AAA-S) and six questions related to demographic details of the respondents. CIPS consists of 20 items that are scored from 1 to 5 , reflecting the degree to which a person feels like an imposter. It is a one-dimensional questionnaire, with the total score expressing the frequency of the imposter phenomenon symptoms (Dudău 2014). A pilot study was tested on ten professionals who were personally known to the researchers. The purpose of the pilot study was to test for face validity, to eliminate ambiguous questions, and to ensure that the questionnaire could be completed without difficulty and within the stated period. No issues were discovered during the pilot study.

\subsection{Data gathering}

The questionnaire was disseminated via e-survey using SurveyMonkey. The electronic requests to participate were sent to a list of potential respondents that was populated primarily by leveraging the networks from the South African Institute of Directors, Businesswomen's Association of South Africa as well as the Black Management Forum. All participants gave their consent in accordance with the ethical standards laid down by the Research Ethics Committee of the institution under whose auspices this research was conducted.

\section{Results}

In all 171 responses were received, but six were discarded as they were incomplete and qualifying data such as data on gender were missing. Of the 165 complete responses received, $58.2 \%$ were received from females, $32.7 \%$ were from white respondents and $67.3 \%$ were from non-white respondents.

\subsection{Construct reliability and validity}

Cronbach's alpha coefficients of .919 and .700 were calculated for the constructs of imposter phenomenon and assertiveness respectively (Nunnally \& Bernstein 1994; Henson 2001), verifying the reliability demonstrated in earlier studies. A principal components analysis (PCA) was performed on both measurement instruments. The suitability of using a PCA was assessed prior to analysis. The overall Kaiser-Meyer-Olkin (KMO) measures were 0.91 and 0.789 respectively. Bartlett's test of sphericity was statistically significant $(p<.005)$ in both instances, indicating that the data were probably factorisable. In respect of the CIPS, a visual inspection of the scree plot indicated that one component should be retained (Table 1). The first component had an eigenvalue of 8.328 and explained $41.64 \%$ of the total variance. A one-factor model was therefore used, following the example of Simon and Choi (2018), who performed a CFA on the CIPS and concluded that a one-factor model was indeed the most appropriate. In respect of the AAA-S, only one factor was found. It had an eigenvalue of 2.526 and explained $49.1 \%$ of the variance. 
Table 1: Scree plot of the CIPS factors

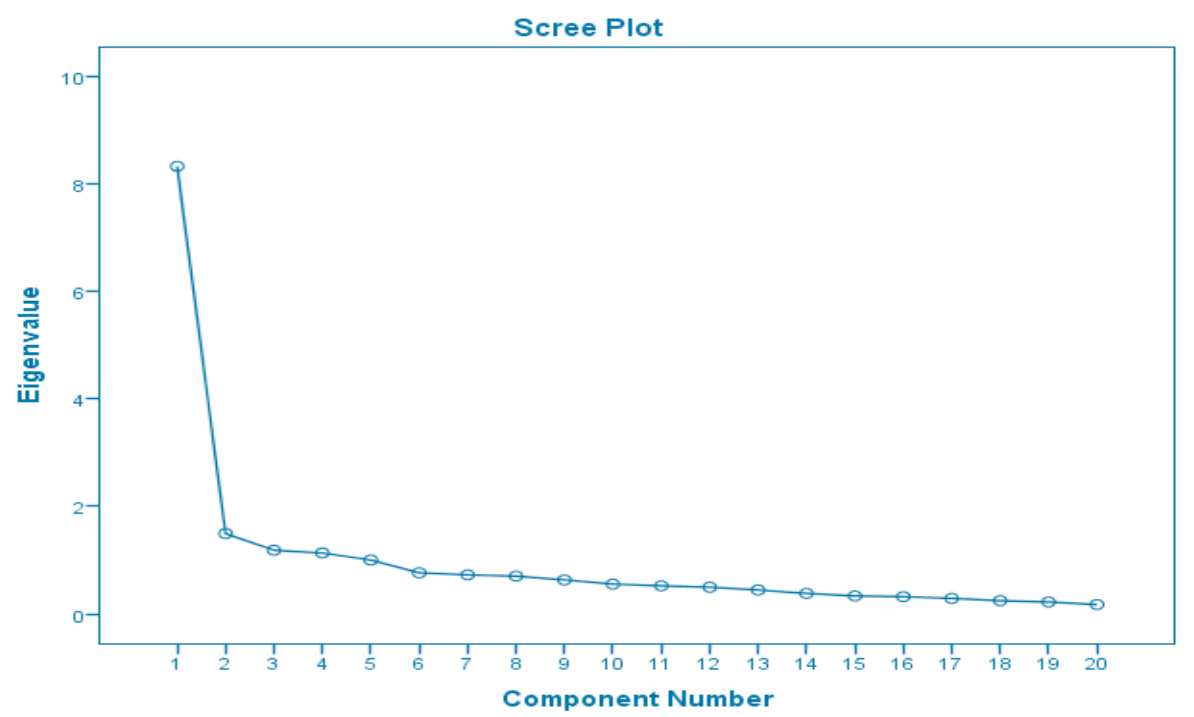

\subsection{Is the imposter phenomenon prevalent in the South African context?}

To determine whether the imposter phenomenon is prevalent among a sample of professionals in South Africa, the researchers calculated the IP scores for each respondent by generating the sum of the components of the CIPS for each respondent. The IP score for the 165 respondents had a mean of 54 and a mode of 59 which, according to Clance et al (1995), falls under the moderate levels of IP. A total of $85.5 \%$ of all the respondents reported at least a moderate level of IP, with $27.9 \%$ reporting either frequent or intense feelings of IP. We can conclude that IP was indeed prevalent among the sample of professionals in South Africa and is worthy of further study.

\subsection{Hypothesis 1}

A hierarchical regression was used to assess the ability of IP to predict assertiveness, after controlling for managerial level and duration of work experience. To assess linearity, a scatterplot of IP against assertiveness with a superimposed regression line was plotted. Visual inspection of these two plots indicated a linear relationship between the variables. There was independence of residuals, as assessed by a Durbin-Watson statistic of 1.858 . There was also homoscedasticity and normality of the residuals. Age, managerial level, and duration of work experience were entered at Step 1, explaining $1.3 \%$ of the variance in assertiveness. After entry of IP at Step 2, the total variance explained by the model as a whole was $10.9 \%, F(4,160)=4.863, p<.001$. Imposter phenomenon accounted for $9.6 \%$ of the explained variability in assertiveness with an adjusted $R^{2}=8.6 \%$, with a significant F-change, $p<.005$ with a medium size effect (Cohen 1988). In the final model, only IP was statistically significant, with a beta coefficient of $-.313, p<.005$. The null hypothesis is therefore rejected and the alternative hypothesis can be accepted. It can be concluded that imposter phenomenon was negatively related to and was a predictor of assertiveness within the sample.

\subsection{Hypothesis 2}

The data file was split between male and female respondents and a simple regression analysis was run to test the relationship between IP and assertiveness within the separate groups.

The linear regression for females established that the imposter phenomenon was able to predict assertiveness with statistical significance, $F(1,94)=20.941, p<.005$ and that the imposter phenomenon accounted for $18.2 \%$ of the explained variability in assertiveness with adjusted $R^{2}=$ $17.3 \%$, a medium sized effect according to Cohen (1988). A beta coefficient of $-.427, p<.0005$ was also found. The regression equation was IP $=543.307+(-3.473 \times$ assertiveness $)$.

However, a linear regression for males established that imposter phenomenon could not statistically significantly predict assertiveness, $F(1,67)=1.952, p=.167$ and imposter phenomenon accounted for only $2.8 \%$ of the explained variability in assertiveness with adjusted $R^{2}=1.4 \%$. A beta coefficient of $-.168, p=.167$ was also found. The regression equation was: IP $=415.41-1.215$ (assertiveness).

We therefore reject the null hypothesis, accept the alternative hypothesis and conclude that the imposter phenomenon is negatively related to and a predictor of assertiveness in the sample of female professionals but not male professionals taken in a South African context. 


\section{Discussion of results}

The purpose of this study was to investigate whether IP has an effect on assertiveness, particularly in female professionals in South Africa. The survey produced in this study set out to explore the existing literature and assess whether the assumptions from the literature and findings were applicable to a sample of professionals in South Africa.

\subsection{Discussion of descriptive statistics}

The study was carried out to examine the relationship between two variables, namely IP and assertiveness, in professionals in South Africa. According to Shrivastava, Selvarajah, Meyer and Dorasamy (2014), South Africa is a diverse cultural melting pot, as was borne out by the demographic data gathered regarding race, gender and experience levels of the respondents. In a different geographic setting the racial composition of the study would be considered questionable, but given the dynamics in South Africa, the results mirror reality. The most recent census, which was conducted in South Africa in 2011, revealed that the population split by race was $8.9 \%$ white, $8.9 \%$ coloured, $79.2 \%$ black, $2.5 \%$ Indian/Asian and $0.5 \%$ other (Census 2011). The proportion of whites in the sample was higher than national levels. This can be explained by the Commission of Employment Equity report for 2011 to 2012, which reported that white professionals in South Africa occupied $59.1 \%$ of senior management roles within the country's largest employers.

\subsection{Does IP exist in a South African context?}

Limited research on IP has been conducted in Africa so the first question that this research assessed was whether IP did in fact exist among professionals in South Africa. The findings suggest that IP does indeed exist among professionals in South Africa.

Based on the definition of IP by Clance and Imes (1978), the results can be interpreted as indicating that, on average, $85.5 \%$ of professionals in South Africa experience at least a moderate fear of failure, a need to be the best, to be superhuman, feel guilty about their success and believe that their success is the result of luck as opposed to hard work.

\subsection{Discussion of hypothesis 1}

The hypothesis proposed that there is a negative correlation between IP and assertiveness in professionals in South Africa and further that IP is a negative predictor of assertiveness in South African professionals.

DeYoung et al (2013) in their exploration of trait theory describe assertiveness as a trait relating to dominance, drive and leadership and define it as a dimension of extraversion. Earlier research by Chae, Piedmont, Estadt and Wicks (1995) examined the relationship between the big five leadership traits and IP. They looked at extraversion and agreeableness and whether these correlated with IP. Chae et al (1995) found that there is a significant but low negative correlation between Chance's Imposters Phenomenon Scale (CIPS) and extraversion. Later studies by Bernard, Dollinger and Ramaniah (2002) refuted this as their tests were not able to identify a relationship between IP and extraversion.

Furthermore, Viswanatham and Sharma (2013) found that individuals that had lower assertiveness scores were prone to experiencing self-denial, which prevented them from achieving their own goals; this behaviour is in some respects similar to behaviours displayed by imposters, according to Langford and Clance (1993).

Based on the findings of the above literature, this study was conducted to establish whether there is a statistically significant relationship between IP and assertiveness among the professionals assessed in South Africa. The tests were conducted on 165 professionals in South Africa and looked at professionals of all levels, genders and races. A Pearson's correlation test revealed a moderate negative relationship between IP and assertiveness. This meant that as an individual's imposter phenomenon levels increased, his or her assertiveness decreased. Conversely, if the imposter phenomenon levels were low then assertiveness levels were likely to be higher.

\subsection{Discussion of hypothesis 2}

Hypothesis 2 asserted that IP has a greater effect on female professionals than on their male counterparts. Based on the results of hypothesis 1 , where a moderate negative relationship between IP and assertiveness was established, the data file was split to delve deeper into the strength of the relationship and to understand whether IP was a predictor of assertiveness in female professionals in particular. There was a significant linear relationship between IP and assertiveness. This was shown through the $\mathrm{R}^{2}$ of $18.2 \%$, which means that an $18.2 \%$ change in assertiveness could be attributed to IP. This $R^{2}$ could be explained by other antecedents identified in the literature by Dudău (2014) and Li et al 
(2014) that suggest that family origins, societal origins and lack of parental care are predictors of IP. Li et al (2014) add that that the more parental care an individual receives, the less IP feelings they experience but that overprotection leads to high IP scores. Peteet, Montgomery and Weekes (2015) further expanded on the topic, stating that low levels of psychological well-being and ethnic identity are predictors of IP in minority students.

These findings indicate that gender does have a significant effect on the correlation between IP and assertiveness. The variation found between the effect of IP on assertiveness in females as opposed to males supports the findings of Langford and Clance (1993) and Bernard et al (2002), who reported that IP has a greater effect on women as they are limited by a constant battle between feminine and masculine traits such as autonomy and assertiveness.

\section{Conclusion}

This research investigated the relationship between IP and assertiveness in professionals in a South African context. The main aim was to determine whether IP had an effect on levels of assertiveness, specifically in female professionals in South Africa, as previous research on this topic had been limited. The literature suggested that IP was mostly found in women. It also suggested that assertiveness, as a dimension of extraversion, was an important leadership trait that was lacking in female leaders. The literature suggests that if extraversion and IP are negatively correlated, then the same could be assumed for assertiveness. Additional research also shows that one of the reasons why females do not progress as fast as their male counterparts may be their lack of assertiveness in the workplace.

The research findings have provided insights into both the subject of IP and assertiveness:

- The first finding was that IP is indeed prevalent among professionals in South Africa. This research showed that feelings of IP were experienced across all respondents in varying degrees and predominantly at moderate levels. There were no significant findings to suggest that IP levels varied due to race and gender.

- The second finding of the study was that there is a moderate negative correlation between IP and assertiveness, which means that as the levels of IP feelings decrease, assertiveness levels increase and conversely, if assertiveness levels are low then IP levels are likely to be higher.

- The third finding, emanating from the second finding above, was that IP is a predictor of assertiveness. The results showed that assertiveness could account for a $9.9 \%$ variation in feelings of IP. The remaining predictors of IP could then be assumed to be family origin, societal origins and parental care, as suggested by the literature.

- A more detailed analysis in which the data were split across gender gave rise to a fourth finding, namely that the relationship between IP and assertiveness is only significant among female professionals in South Africa.

\section{Recommendations and implications for management}

In a business environment in which most, if not all, corporates are striving for diversity, it is necessary to undertake research to ascertain why imbalances still exist and how to address them. This research aimed to uncover the relationship between IP and assertiveness, particularly among women, and was able to show that the relationship between IP and assertiveness across gender differs significantly, indicating that higher levels of IP lead to lower levels of assertiveness among professional women in South Africa.

Howe-Walsh and Turnbull's (2016) research suggests that women who tend to have lower levels of self-belief may create barriers for themselves regarding their career growth and development. Women in their study displayed a sense of not being good enough and despite being high achievers found themselves doubting their abilities. Human resource and other leadership functions should therefore take proactive measures to explicitly demonstrate to female leaders in their organisation that they do indeed belong in their posts and deliver value to the organisation. The finding that IP does play a part in holding women back contributes to our understanding of the reasons why we have a limited number of women in senior executive roles. Awareness of the hegemonic masculinity underlying dominant social practices may well help women to overcome their sense of IP (Gherardi \& Poggio 2001). Tarr-Whelan (2009) provides practical steps that will enable women to fulfil their potential and overcome the stereotyping and biases they face in the workplace, and thus to reduce their feelings of being an imposter.

\subsection{Limitations of research and suggestions for future research}

As self-reporting measures were used, there may be an element of bias in the reporting by respondents, in particular ones who do display IP, as they may underplay their achievements (Clance \& Imes 1978). Thompson and Berenbaum (2011) state that one limitation of the AAA-S is that it does not measure all forms of assertiveness, only two direct forms of assertiveness. An investigation to 
determine whether there is a difference in IP levels across the various life stages would provide an interesting addition to the literature on IP. The influence of other individual factors, such as level and type of education, as well as contextual factors such as the culture of the organisation, may be considered. Further studies could be conducted to determine whether IP does exist or has implications within the informal sectors in Africa. This study was specifically focused on professionals in South Africa employed in the formal sector. It would be interesting to see whether the results would be the same if the informal sector had been targeted. Furthermore, are there other psychological factors that prevent women from progressing in their careers and are there moderating variables that affect the relationship between IP and assertiveness?

Given that South Africa needs to grow its economy to start to address the challenges of unemployment, poverty and associated problems, it is important that we draw on all the talent available to fill managerial and leadership roles. Men still dominate these ranks and South African organisations should provide opportunities for women to take up their rightful roles alongside men in the leadership ranks. Understanding the impact of the imposter phenomenon and its effect on assertiveness, particularly among women, will contribute to achieving this objective.

\section{List of references}

Ames, DR \& Flynn, FJ. 2007. What breaks a leader: The curvilinear relation between assertiveness and leadership. Journal of Personality and Social Psychology, 92(2):307.

Arena, D \& Page, N. 1992. The imposter phenomenon in the clinical nurse specialist role. Image The Journal of Nursing Scholarship, 24(2):121-125.

Bernard, NS, Dollinger, SJ \& Ramaniah, NV. 2002. Applying the big five personality factors to the impostor phenomenon. Journal of Personality Assessment, 78(2):321-333.

Bernat, E. 2008. Towards a pedagogy of empowerment: The case of "impostor syndrome" among pre-service non-native speaker teachers in TESO. ELTED, 11:1-8.

Braidford, P, Stone, I \& Tesfaye, B. 2013. Gender, disadvantage and enterprise support - lessons from women's business centres in North America and Europe. Journal of Small Business and Enterprise Development, 20(1):143-164.

Statistics South Africa. 2012. Census 2011: Census in brief. Available at: ISBN 978-0-621-41388-5 (accessed on 25 November 2015).

Chae, JH, Piedmont, RL, Estadt, BK \& Wicks, RJ. 1995. Personological evaluation of Clance's Imposter Phenomenon Scale in a Korean sample. Journal of Personality Assessment, 65(3):468-485.

Clance, PR. 1985. The impostor phenomenon: Overcoming the fear that haunts your success. Georgia: Peachtree Publisher Ltd.

Clance, PR, Dingman, D, Reviere, SL \& Stober, DR. 1995. Impostor phenomenon in an interpersonal/social context: Origins and treatment. Women \& Therapy, 16(4):79-96.

Clance, PR \& Imes, SA. 1978. The imposter phenomenon in high achieving women: Dynamics and therapeutic intervention. Psychotherapy: Theory, Research \& Practice, 15(3):1-8.

Clark, M, Vardeman, K, \& Barba, S. 2014. Perceived inadequacy: A study of the imposter phenomenon among college and research librarians. College \& Research Libraries, 75(3):255-271.

Cohen, J. 1988. Statistical power analysis for the behavioral sciences. $2^{\text {nd }}$ edition. New York, NY: Psychology Press.

Cokley, K, McClain, S, Enciso, A \& Martinez, M. 2013. An examination of the impact of minority status stress and impostor feelings on the mental health of diverse ethnic minority college students. Journal of Multicultural Counseling and Development, 41(2):82-95.

Cook, A \& Glass, C. 2014. Women and top leadership positions: Towards an institutional analysis. Gender, Work \& Organization, 21(1):91-103.

Cusack, CE, Hughes, JL \& Nuhu, N. 2013. Connecting gender and mental health to imposter phenomenon feelings. Psi Chi Journal of Psychological Research, 182.

DeYoung, CG, Weisberg, YJ, Quilty, LC \& Peterson, JB. 2013. Unifying the aspects of the Big Five, the interpersonal circumplex, and trait affiliation. Journal of Personality, 81(5):465-475.

Dudău, DP. 2014. The relation between perfectionism and impostor phenomenon. Procedia-Social and Behavioral Sciences, 12(7):129-133. 
Eagly, AH \& Carli, LL. 2007. Through the labyrinth: The truth about how women become leaders. Harvard Business Press.

Eleftheriou-Smith, L. 2015. Bic apologises for sexist 'Think like a man' advert designed to celebrate Women's Day. Available at: http://www.independent.co.uk/news/world/bic-apologises-for-sexist-thinklike-a-man-advert-designed-to-celebrate-south-africa-womens-day-10449842.html (accessed: 20 January 2016).

Ely, RJ \& Meyerson, DE. 2000. Theories of gender in organizations: A new approach to organizational analysis and change. Research in Organizational Behavior, 22:103-151.

Fried-Buchalter, S. 1997. Fear of success, fear of failure, and the imposter phenomenon among male and female marketing managers. Sex Roles, 37(11):847-859.

Gherardi, S \& Poggio, B. 2001. Creating and recreating gender order in organizations. Journal of World Business, 36(3):245-259.

Heilman, ME. 2001. Description and prescription: How gender stereotypes prevent women's ascent up the organizational ladder. Journal of Social Issues, 57(4):657-674.

Henson, RK. 2001. Understanding internal consistency reliability estimates: A conceptual primer on coefficient alpha. Measurement and Evaluation in Counseling and Development, 34(3):177.

Howe-Walsh, L \& Turnbull, S. 2016. Barriers to women leaders in academia: Tales from science and technology. Studies in Higher Education, 41(3):415-428.

Kwan, V. 2015. Asian Americans and the impostor phenomenon. Doctoral thesis. San Francisco State University.

Langford, J \& Clance, PR. 1993. The imposter phenomenon: Recent research findings regarding dynamics, personality and family patterns and their implications for treatment. Psychotherapy: Theory, Research, Practice, Training, 30(3):495.

Lazarus, AA. 1973. On assertive behavior: A brief note. Behavior Therapy, 45:697-699. Available at: doi:10.1016/S0005-78947380161-3.

Li, S, Hughes, JL \& Thu, SM. 2014. The links between parenting styles and imposter phenomenon. Psi Chi Journal of Psychological Research, 192.

Linderman, D, Britton, D \& Zundi, E. 2016. I don't know why they make it so hard here: Institutional factors and undergraduate women's STEM participation. International Journal of Gender, Science and Technology, 8(2):221-241.

Lounsbury, JW, Sundstrom, ED, Gibson, LW, Loveland, JM \& Drost, AW. 2016. Core personality traits of managers. Journal of Managerial Psychology, 31(2):434-450.

Mall, F. 2015. JSE-listed corporations continue without adequate representation of women. Available at: http://www.bwasa.co.za/news/bwa-women-in-leadership-census-media-release (accessed on 25 January 2016).

Mattie, C, Gietzen, J, Davis, S \& Prata, J. 2008. The imposter phenomenon: Self-assessment and competency to perform as a physician assistant in the United States. The Journal of Physician Assistant Education, 19(1):5-12.

McDowell, WC, Grubb III, WL \& Geho, PR. 2015. The impact of self-efficacy and perceived organizational support on the imposter phenomenon. American Journal of Management, 15(3):23.

Neureiter, M \& Traut-Mattausch, E. 2016. An inner barrier to career development: Preconditions of the impostor phenomenon and consequences for career development. Frontiers in Psychology, 7.

Neureiter, M \& Traut-Mattausch, E. 2016. Inspecting the dangers of feeling like a fake: An empirical investigation of the impostor phenomenon in the world of work. Frontiers in Psychology, 7.

Nunnally, JC \& Bernstein, IH. 1994. The assessment of reliability. Psychometric Theory, 31:248-292.

Parham, JB, Lewis, CC, Fretwell, CE, Irwin, JG \& Schrimsher, MR. 2015. Influences on assertiveness: Gender, national culture, and ethnicity. Journal of Management Development, 34(4):421-439.

Peteet, BJ, Brown, CM, Lige, QM \& Lanaway, DA. 2015. Impostorism is associated with greater psychological distress and lower self-esteem for African American students. Current Psychology, 34(1):154-163.

Peteet, BJ, Montgomery, L \& Weekes, JC. 2015. Predictors of imposter phenomenon among talented ethnic minority undergraduate students. The Journal of Negro Education, 84(2):175-186. 
Pfafman, TM \& McEwan, B. 2014. Polite women at work: Negotiating professional identity through strategic assertiveness. Women's Studies in Communication, 37(2):202-219.

Powell, GN. 2012. Six ways of seeing the elephant: The intersection of sex, gender, and leadership. Gender in Management: An International Journal, 27(2):119-141.

Rohrmann, S, Bechtoldt, M \& Leonhardt, M. 2016. Validation of the impostor phenomenon among managers. Frontiers in Psychology, 7:821.

Rosette, AS, Koval, CZ, Ma, A \& Livingston, R, 2016. Race matters for women leaders: Intersectional effects on agentic deficiencies and penalties. The Leadership Quarterly, 27(3):429-445.

Sakulku, J \& Alexander, J. 2011. The impostor phenomenon. International Journal of Behavioral Science (IJBS), 6(1).

Saunders, M, Lewis, P, \& Thornhill, A. 2012. Research methods for business students. $6^{\text {th }}$ edition. India: Pearson Education.

Shrivastava, S, Selvarajah, C, Meyer, D \& Dorasamy, N. 2014. Exploring excellence in leadership perceptions amongst South African managers. Human Resource Development International, 17(1):4766.

Schubert, N \& Bowker, A. 2017. Examining the impostor phenomenon in relation to self-esteem level and self-esteem instability. Current Psychology, 1-7.

Simmons, D. 2016. Imposter syndrome, a reparative history. Engaging Science, Technology and Society, 2:106-127.

Simon, M \& Choi, YJ. 2018. Using factor analysis to validate the Clance Impostor Phenomenon Scale in a sample of science, technology, engineering and mathematics doctoral students. Personality and Individual Differences, 12(1):173-175.

Stein, SJ \& Book, H. 2010. The EQ edge: Emotional intelligence and your success, 25. Wiley.

Tarr-Whelan, L. 2009. Women lead the way: Your guide to stepping up to leadership and changing the world. San Francisco, California: Berrett-Koehler Publishers Inc.

Thompson, RJ \& Berenbaum, H. 2011. Adaptive and aggressive assertiveness scales AAA-S. Journal of Psychopathology and Behavioral Assessment, 33(3):323-334.

Vergauwe, J, Wille, B, Feys, M, De Fruyt, F \& Anseel, F. 2015. Fear of being exposed: The traitrelatedness of the impostor phenomenon and its relevance in the work context. Journal of Business and Psychology, 30(3):565-581.

Viswanatham, KN \& Sharma, S. 2013. Subjective well-being in high assertive women managers. ASCI Journal of Management, 43(1):29-38.

Woetzel, J. 2015. The power of parity: How advancing women's equality can add $\$ 12$ trillion to global growth. eSocialSciences. 